\title{
Augmented expression of polo-like kinase 1 indicates poor clinical outcome for breast patients: a systematic review and meta-analysis
}

\author{
Yunfeng Zhang ${ }^{1 *}$, Zhibin Wu ${ }^{2 *}$, Dapeng Liu ${ }^{1 *}$, Meng Wang ${ }^{1}$, Guodong Xiao ${ }^{1}$, Peili \\ Wang $^{1}$, Xin Sun ${ }^{1}$, Hong Ren ${ }^{1}$, Shou-Ching Tang ${ }^{3,4}$ and Ning Du ${ }^{1}$ \\ ${ }^{1}$ Department of Thoracic Surgery and Oncology, the Second Department of Thoracic Surgery, Cancer Center, the First \\ Affiliated Hospital of Xi'an Jiaotong University, Xi'an, Shaanxi Province, 710061, China. \\ 2 The Third Department of Surgery, Shenmu County Hospital, Yulin, Shaanxi Province, 719300, China. \\ ${ }^{3}$ Georgia Cancer Center Medical College of Georgia, Augusta University, Augusta, GA, 30912, United States. \\ ${ }^{4}$ Tianjin Medical University Cancer Institute and Hospital, Tianjin 300060, China. \\ * These authors have contributed equally to this work \\ Correspondence to: Ning Du, email: andrewdu@126.com
}

Shou-Ching Tang, email: STANG@augusta.edu

Keywords: polo-like kinas 1, breast cancer, clinical outcome, meta-analysis

Received: February 06, $2017 \quad$ Accepted: March 09, $2017 \quad$ Published: April 20, 2017

Copyright: Zhang et al. This is an open-access article distributed under the terms of the Creative Commons Attribution License 3.0 (CC BY 3.0), which permits unrestricted use, distribution, and reproduction in any medium, provided the original author and source are credited.

\section{ABSTRACT}

Polo-like kinases 1 (PLK1), a key regulator of mitosis, plays an essential role in maintaining genomic stability. Up-regulation of PLK1 was found in tumorigenesis and tumor progression of diverse cancers. However, the clinicopathological and prognostic implications of PLK1 in breast cancer (BC) have yet to be unveiled. Therefore, using PubMed, Web of Science, Embase, and Chinese databases, we conducted a metaanalysis to define the potential clinical value of PLK1 in BC. Eleven eligible articles with 2481 patients enrolled were included in the present meta-analysis, of which eight studies reported on the relationship between PLK1 expression and clinicopathological features, and nine studies provided survival data in BC patients. Furthermore, the results revealed that high PLK1 levels were significantly associated with larger tumor size ( $O R=1.703,95 \% C I s: 1.315-2.205, P<0.001)$, higher pathological grading (OR=6.028, 95\%CIs: 2.639-13.772, $P<0.001$ ), and lymph node metastasis (OR= 1.524, 95\%CIs: 1.192-1.950, $P=0.001)$. Moreover, PLK1 was found to be a valuable factor for distinguishing lobular BC from ductal BC with the pooled $O R=0.215(95 \% C I s$ : $0.083-0.557, P=0.002)$. Analysis of included data showed that high PLK1 expression significantly indicated worse overall survival for BC patients $(H R=3.438,95 \%$ CIs: 2.293-5.154, $P<0.001$ ), as well as worse cancer specific survival and disease-free survival (HR=2.414, 95\%CIs: 1.633-3.567, $P<0.001$ and $H R=2.261,95 \% C I s: 1.796-$ 2.951, $P<0.001$, respectively). This quantitative meta-analysis suggests that high PLK1 expression is a credible indicator for the progression of $B C$ and confirms a higher risk of a worse survival rate in patients with BC.

\section{INTRODUCTION}

Breast cancer $(\mathrm{BC})$, the most prevalent cancer overall among women, represents approximately 1.7 million new cancer cases worldwide annually [1]. Due to significant progress made in current treatment, the survival rates of $\mathrm{BC}$ patients have increased in developed regions. However, the efficacy of the diagnostic and therapeutic techniques for $\mathrm{BC}$ is still limited because of the multi-gene aberrations and complex biological mechanism of this disease. Currently, surgery is still the cornerstone for $\mathrm{BC}$ treatment, in conjunction with chemotherapy, radiotherapy, and hormone therapy [2]. Advances in the sequencing of the human genome have 
facilitated the understanding of the underlying molecular mechanisms of BC heterogeneity [3]. High-throughput molecular profiling has also contributed to a paradigm shift towards increasingly targeted therapy. Detection of proteins and genes, such as estrogen receptors (ERs) [4], vascular endothelial growth receptors (VEGRs) [5] and transcription factor nuclear factor-kappa B (NF-kB) [6], involved in $\mathrm{BC}$ development at the molecular level could provide insight into the molecular and genetic heterogeneity of BC. Unfortunately, these factors could not clearly unveil the nature of biological changes in BC. Thus, new strategies are needed to explore novel biomarkers for $\mathrm{BC}$ to achieve the goal of individualized approaches to treatment.

Polo-like kinase 1 (PLK1), the best-characterized member of polo-like kinase family, plays a crucial role in cell-cycle regulation via maintaining genome stability [7]. Previous studies showed that PLK1 is involved in the regulation of DNA damage repair by mediating checkpoint kinase 2 (Chk2) and the scaffold protein claspin [8]. Given the crucial role of PLK1 in cell-cycle regulation and DNA damage repair, it is not surprising that it is overexpressed in a variety of malignant neoplasms such as non-small cell lung cancer, bladder cancer, and colorectal cancer [9, 10]. Current studies demonstrated that high expression of PLK1 correlates with poor survival for the patients of gastric cancer and neuroblastomas $[11,12]$. However, studies about the clinicopathological and prognostic significance of PLK1 in BC are comparatively few. Thus, we conducted a meta-analysis and systematic review of published literature to investigate the clinicopathological and prognostic implications of PLK1 expression in BC patients.

\section{RESULTS}

\section{Studies inclusion}

Figure 1 shows the flowchart for this metaanalysis. Our search of literature yielded 1042 articles for consideration. After title and abstract evaluation, 56 articles were identified in terms of PLK1 expression in BC patients for further work. Finally, a total of 11 studies enrolling $2481 \mathrm{BC}$ patients ranging from 2005 to 2016 were included [13-23], of which eight studies $[13,15-18,21-23]$ reported clinicopathological data and nine studies $[13,14,16-22]$ provided prognostic data. The NOS scores of included studies varied from 6 to 9 , of which two studies [14, 19] were assesses as 9 scores and two studies $[16,21]$ were evaluated as 8 scores, as well as 5 studies [13, 17, 18, 20, 22] with 7 scores and one [23] with 6 scores. In total, eight English studies [13, $14,16,17,19-22]$ and three Chinese studies [15, 18, 23] were included in our analysis, of which the sample sizes ranged from 32 to 979. Specifically, eight studies [13, 15, 16, 18, 21-23] including 1779 patients reported on the relationship between PLK1 and patient age; seven [13, 15-17, 21-23] provided sufficient pathological grading information for 1989 BC patients; seven [13, 15-18, 21-23] provided sufficient lymph node information for 2110 BC patients, and three studies with 1129 patients provided $[13,15,22]$ sufficient tumor type information. Regarding crucial biomarkers for BC, studies detecting the association between PLK1 and ER status (seven studies with 1155 patients) $[15-18,21-23]$, PR status (five studies with 1745 BC patients) [13, 15, 17, 21-23], HER2 status (four studies $1240 \mathrm{BC}$ patients) $[13,15,17,22]$, and p53 mutation status (three studies including 713 patients) [16, $17,21]$ were included. Four studies [17-19, 22] exploring the prognostic role of PLK1 for OS in BC patients, as well as three studies $[13,14,21]$ for CSS and five studies [14, $16,17,19,20]$ for DFS. Three studies [14, 18, 19] directly provided the multivariate $\mathrm{HR}$ and their 95\% CIs; three studies [13, 19, 20] reported univariate HRs; the other five studies did not present estimated HRs; however, we obtained two HRs based on original data in two studies $[16,21]$ and remaining 3 studies' HRs [14, 17, 22] were derived from their respective survival curves. See Table 1 for further detailed characteristics of the included studies.

\section{Correlation of PLK1 expression and clinicopathological factors of BC patients}

Results of the meta-analysis indicated that high PLK1 expression significantly correlated with large tumor size (tumor size $>2 \mathrm{~cm}$ ) with low heterogeneity $(\mathrm{OR}=$ 1.703 , 95\% CIs: $1.315-2.205, P<0.001 ; \mathrm{I}^{2}=30.10 \%, P$ $=0.198$, Figure $2 \mathrm{a}$ ) in six studies with $1779 \mathrm{BC}$ patients; higher tumor grade $(\mathrm{OR}=6.028,95 \%$ CIs: 2.639-13.772, $P<0.001$, Figure 2b) in 1989 BC patients; and lymph node metastasis $(\mathrm{OR}=1.524,95 \%$ CIs: $1.192-1.950, P=$ 0.001 , Figure 2c) in 1975 BC patients.

Also, PLK1 was found to be able to distinguish lobular BC from ductal BC with the pooled OR of 0.215 (95\% CIs: 0.083-0.557, $P=0.002$, Figure 2d). However, there is no significance associated between PLK1 expression and age $(\mathrm{OR}=1.018,95 \%$ CIs: 0.795-1.303, $P=0.888)$. Next, we provided evidence that elevated PLK expression was negatively associated with ERpositive status with high heterogeneity in 7 studies $(\mathrm{OR}=$ 0.392, 95\% CIs: 0.202-0.762, $P=0.006 ; \mathrm{I}^{2}=73.30 \%, P$ $<0.001$, Figure 2e). The pooled OR in 3 studies with 713 $\mathrm{BC}$ patients also indicated a strong relationship between elevated PLK1 expression and p53 mutation status (OR $=6.663,95 \%$ CIs: 4.249-10.448, $P<0.001$, Figure 2f). The pooled ORs did not indicate a significant association between high PLK1 expression with neither PR status nor HER2 status $(\mathrm{OR}=0.560,95 \%$ CIs: 0.229-1.364, $P=0.202$ and $\mathrm{OR}=1.447,95 \%$ CIs: $0.795-2.633, P=$ 
Table 1: Characteristics of studies included in the meta-analysis.

\begin{tabular}{|l|c|l|c|c|l|l|l|l|}
\hline Author & Year & Region & Patients(n) & Cutoff value & Sample & Assay & Score & Data \\
\hline Weichert & 2005 & Germany & 135 & 6(IRS scores) & tissue & IHC & 7 & Both* \\
\hline Miller & 2005 & Sweden & 250 & 6.06 & tissue & RT-PCR & 8 & Both \\
\hline Ivshina & 2006 & Singapore & 249 & 6.08 & tissue & RT-PCR & 8 & Both \\
\hline Han & 2007 & China & 32 & 6 & tissue & IHC & 6 & Clinicopathological imformation \\
\hline Loddo & 2009 & UK & 167 & 0.142 & tissue & IHC & 9 & Prognostic imformation \\
\hline Li & 2009 & China & 248 & 1 & tissue & IHC & 6 & Clinicopathological imformation \\
\hline Li & 2011 & China & 84 & NA* & tissue & RT-PCR & 7 & Both \\
\hline Ali & 2012 & UK & 979 & 2 & tissue & IHC & 7 & Both \\
\hline Maire & 2012 & France & 39 & 3.94 & tissue & RT-PCR & 7 & Prognostic imformation \\
\hline King & 2012 & UK & 215 & 3 & tissue & IHC & 7 & Both \\
\hline Donizy & 2016 & Poland & 83 & 8 & tissue & IHC & 9 & Prognostic imformation \\
\hline
\end{tabular}

NA: not available, Both: study both with clinicopathological and prognostic information.

Table 2: Main results for meta-analysis between PLK1 and clinicopathological features in breast cancer

\begin{tabular}{|c|c|c|c|c|c|c|c|c|c|}
\hline \multirow{2}{*}{$\begin{array}{l}\text { Clinicopathological } \\
\text { features }\end{array}$} & \multirow{2}{*}{$\operatorname{Study}(n)$} & \multirow{2}{*}{ Pooled OR(95\%CIs) } & \multirow{2}{*}{$\mathbf{z}$} & \multirow{2}{*}{$P$} & \multicolumn{3}{|c|}{ Heterogeneity } & \multicolumn{2}{|c|}{\begin{tabular}{|l} 
Publication bias \\
\end{tabular}} \\
\hline & & & & & $I^{2}$ & $P$ & Estimated method & $z$ & $P$ \\
\hline Age & 7 & $1.018(0.795,1.303)$ & 0.14 & 0.888 & $0.00 \%$ & 0.670 & Fixed model & 0.90 & 0.368 \\
\hline Tumor size & 7 & $1.703(1.315,2.205)$ & 4.04 & $<0.001$ & $30.10 \%$ & 0.198 & Fixed model & 0.90 & 0.368 \\
\hline Grading & 7 & $6.028(2.639,13.772)$ & 4.26 & $<0.001$ & $78.90 \%$ & $<0.001$ & Ramdon model & 0.90 & 0.368 \\
\hline Lymph node & 7 & $1.524(1.192,1.950)$ & 3.35 & 0.001 & $43.20 \%$ & 0.090 & Fixed model & 0.62 & 0.536 \\
\hline Tumor type & 3 & $0.215(0.083,0.557)$ & 3.17 & 0.002 & $0.00 \%$ & 0.609 & Fixed model & 0.00 & 1.000 \\
\hline ER status & 7 & $0.392(0.202,0.762)$ & 2.76 & 0.006 & $73.30 \%$ & 0.001 & Ramdon model & 1.50 & 1.330 \\
\hline PR status & 5 & $0.560(0.229,1.364)$ & 1.28 & 0.202 & $76.90 \%$ & 0.002 & Ramdon model & -0.24 & 1.000 \\
\hline P53 & 3 & $6.663(4.249,10.448)$ & 8.26 & $<0.001$ & $22.60 \%$ & 0.275 & Fixed model & 0.00 & 1.000 \\
\hline HER2 & 4 & $1.447(0.795,2.633)$ & 1.21 & 0.226 & $0.00 \%$ & 0.729 & Fixed model & -0.34 & 1.000 \\
\hline
\end{tabular}

Table 3: Summary table of HRs and their $95 \%$ CI for survival analysis

\begin{tabular}{|c|c|c|c|c|}
\hline Survival & HR(95\%CIs) & Significance & Method & Publication bias \\
\hline \multicolumn{5}{|l|}{$\mathrm{OS}$} \\
\hline Weichert2005 & $2.010(0.880,4.590)$ & NS & Survival curve & \\
\hline Loddo2009 & $3.460(1.370,8.710)$ & Poor & Univariate & \\
\hline Li 2011 & $4.760(1.341,6.123)$ & Poor & Multivariate & \\
\hline King 2012 & $3.890(1.820,8.320)$ & Poor & Survival curve & \\
\hline Combined HR & $3.438(2.293,5.154)$ & $\mathrm{z}=5.98, P<0.001 ; I^{2}=0 \%, P=0.487$ & Fixed-effects model & $\mathrm{z}=1.02, P=0.308$ \\
\hline \multicolumn{5}{|l|}{ CSS } \\
\hline Miller2005 & $1.739(1.014,2.985)$ & Poor & Original data & \\
\hline Ali 2012 & $2.600(1.300,5.200)$ & Poor & Univariate & \\
\hline Donizy 2016 & $6.130(2.300,16.330)$ & Poor & Multivariate & \\
\hline Combined HR & $2.414(1.633,3.567)$ & $\mathrm{z}=4.42, P<0.001 ; P^{2}=59.50 \%, P=0.085$ & Fixed-effects model & $\mathrm{z}=1.04, P=0.296$ \\
\hline \multicolumn{5}{|l|}{ DFS } \\
\hline Ivshina 2006 & $1.736(1.378,2.646)$ & Poor & Original data & \\
\hline Loddo2009 & $3.310(1.570,6.970)$ & Poor & Multivariate & \\
\hline Maire 2012 & $3.410(1.030,11.260)$ & Poor & Univariate & \\
\hline King 2013 & $6.050(2.130,17.170)$ & Poor & Survival curve & \\
\hline Donizy 2016 & $3.620(1.500,8.740)$ & Poor & Survival curve & \\
\hline Combined HR & $2.261(1.732,2.951)$ & $\mathrm{z}=6.00, P<0.001 ; I^{2}=52.9 \%, P=0.075$ & Fixed-effects model & $\mathrm{z}=0.73, P=0.462$ \\
\hline
\end{tabular}


0.226, Table 2). All the pooled ORs for the association between PLK1 expression and clinicopathological factors are shown in Table 2.

\section{Prognostic value of PLK1 in BC patients}

To gain insight into the prognostic role of PLK1 in $\mathrm{BC}$, we next investigated the association between PLK1 expression and OS, CSS, and DFS for BC. Consequently, a valuable prognostic effect of PLK1 for poorer OS was found in four studies with a total of $601 \mathrm{BC}$ patients (HR $=3.438,95 \%$ CIs: 2.293-5.154, $P<0.001, \mathrm{I}^{2}=0 \%, P$ $=0.487)$. The pooled HRs of three studies, including 1312 BC patients, also revealed the predictive effect of high PLK1 expression on shorter CSS (HR $=2.414,95 \%$ CIs: 1.633-3.567, $P<0.001 ; \mathrm{I}^{2}=59.5 \%, P=0.085$,). Meanwhile, high PLK expression also presented an unfavorable factor for DSS in the five studies $(\mathrm{HR}=$ 2.261, 95\% CIs: $1.796-2.951, P<0.001 ; \mathrm{I}^{2}=52.9 \%, P$ $=0.075)$. We did not conduct a subgroup analysis due to the heterogeneity not being obvious. The combined HRs for the survival analysis are presented in Table 3 and in Figure 3.

\section{Test of heterogeneity}

In this meta-analysis, we found that there is also obvious heterogeneity in the studies evaluating the association between PLK1 expression and ER status $\left(\mathrm{I}^{2}\right.$ $=73.30 \%, P=0.001 \%)$, as well as PR status $\left(\mathrm{I}^{2}=76.9 \%\right.$, $P=0.002)$. Significant heterogeneity existed in the correlations between high PLK1 expression and tumor grading $\left(\mathrm{I}^{2}=78.90 \%, P<0.0001\right)$. Additionally, studies investigating the prognostic value of PLK1 expression did not present obvious heterogeneity $\left(\mathrm{I}^{2}=0 \%\right.$ for OS, $P=$ $0.487 ; \mathrm{I}^{2}=59.5 \%$ for CSS, $P=0.085$; and $\mathrm{I}^{2}=52.9 \%$ for DFS, $P=0.075)$. The results of the test of heterogeneity for all the analyses are shown in Table 2 and Table 3.

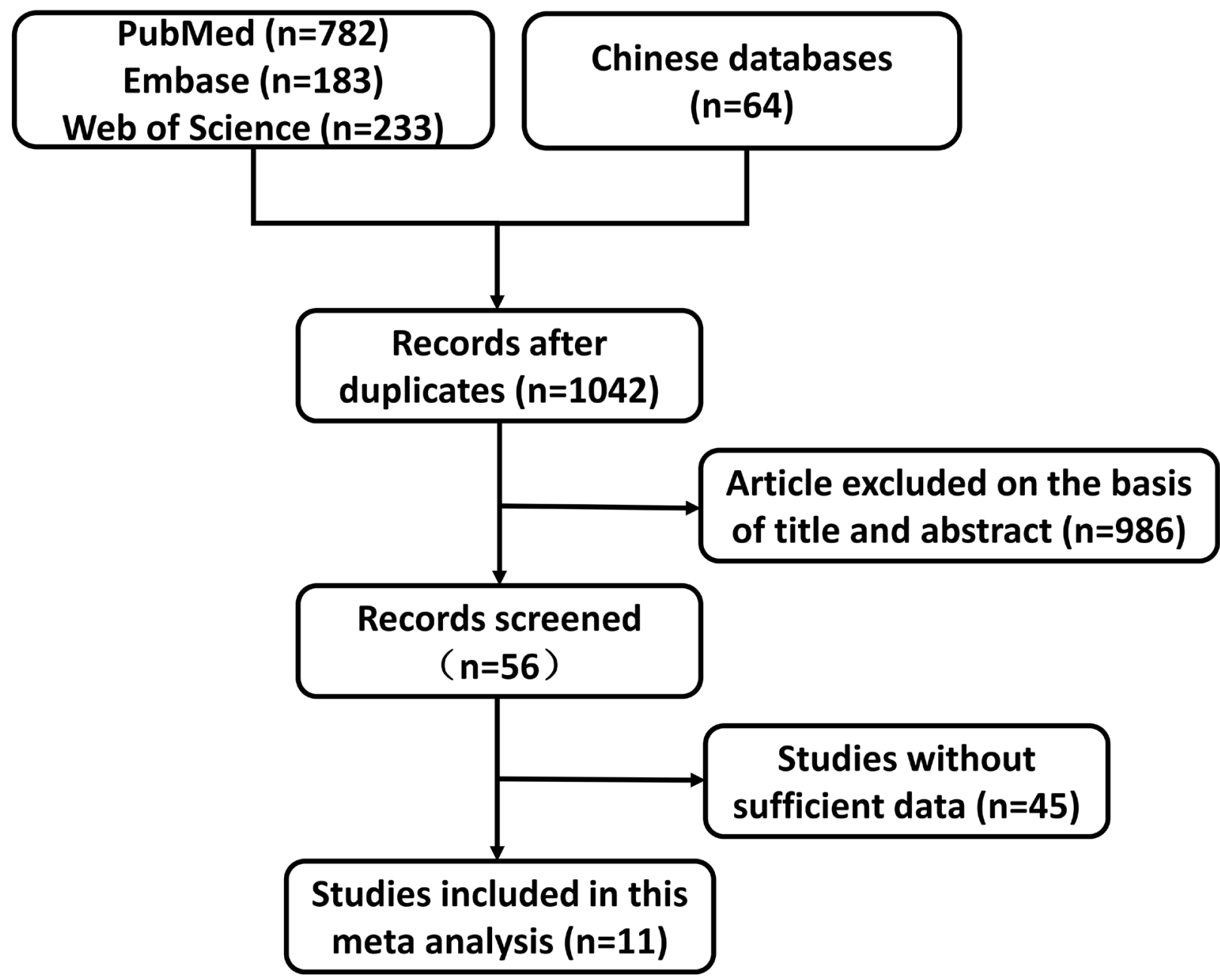

Figure 1: Flowchart of the study selection. 


\section{Publication bias}

As shown in funnel plot, there is no obvious

\section{A}

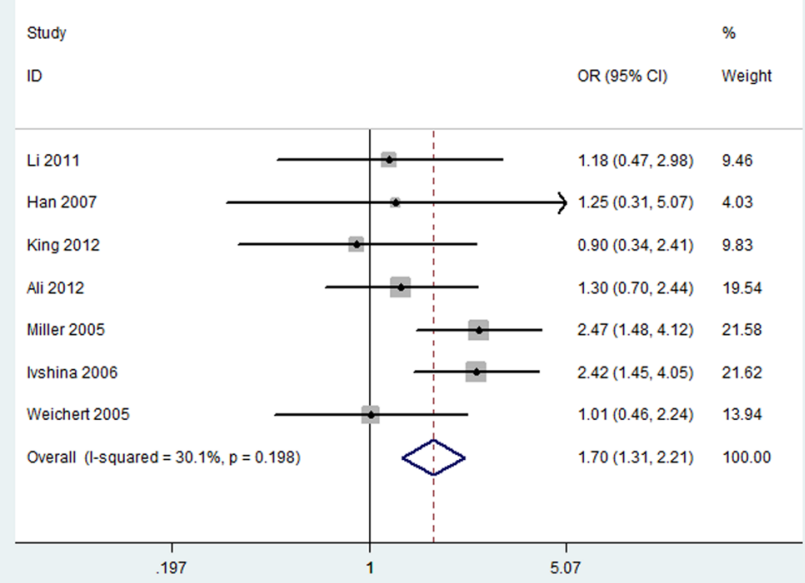

C

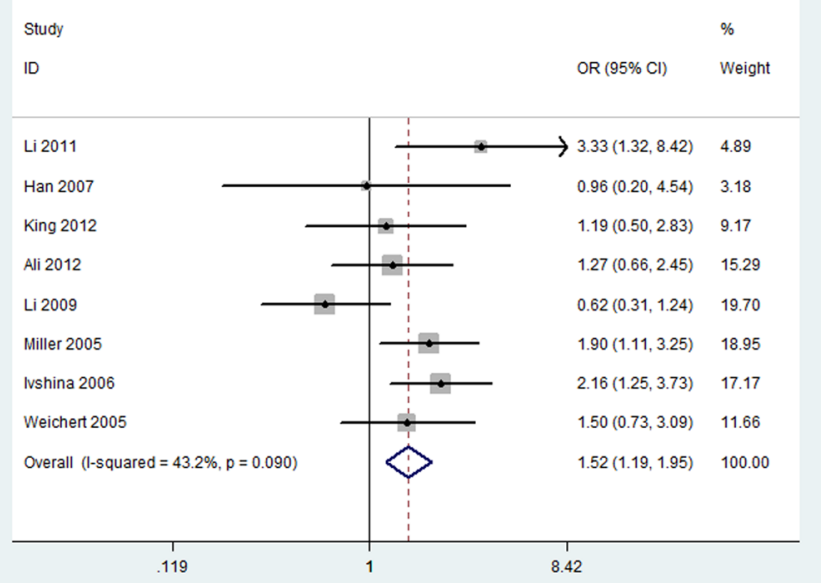

$\mathbf{E}$

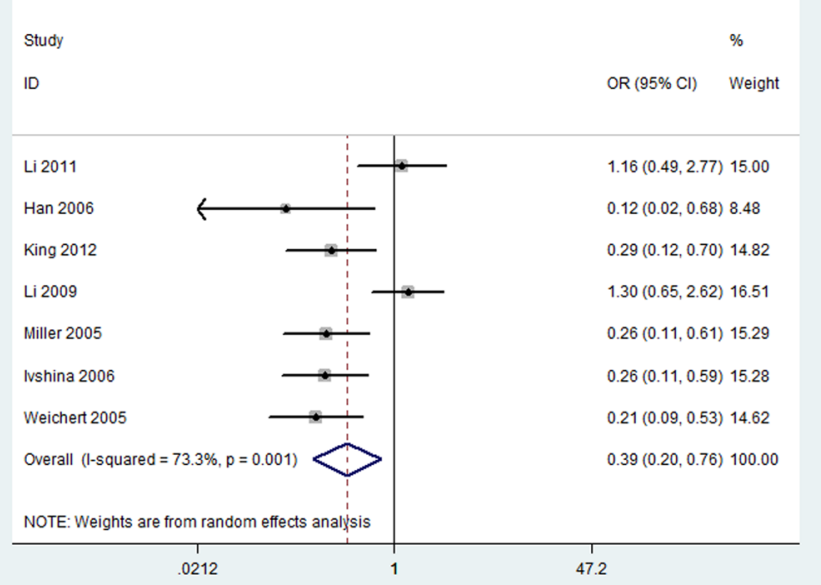

publication bias among all the analysis of PLK1 expression and clinicopathological parameters of $\mathrm{BC}$ patients (Figure 4). The results of Begg' test for each analysis were also presented in Table 2. Nevertheless, the funnel plots and

\section{B}

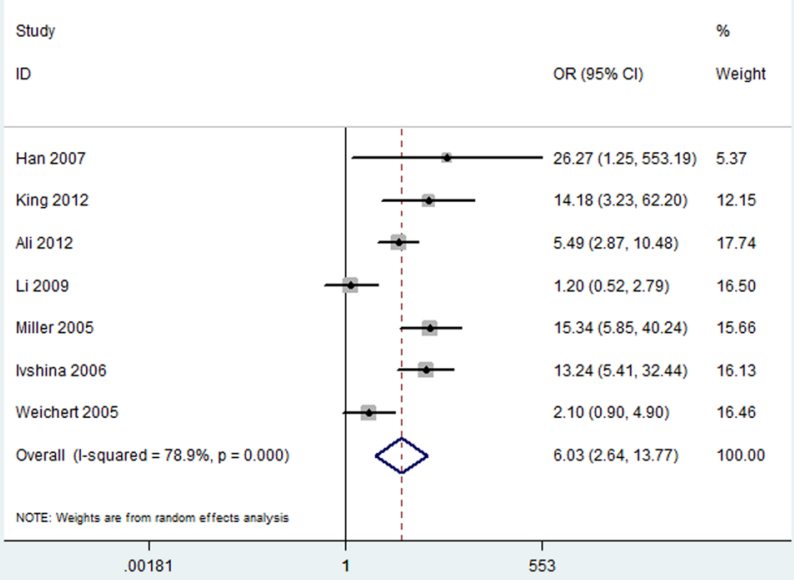

D

Study

ID $\quad$ OR $(95 \% \mathrm{Cl}) \quad$ Weight

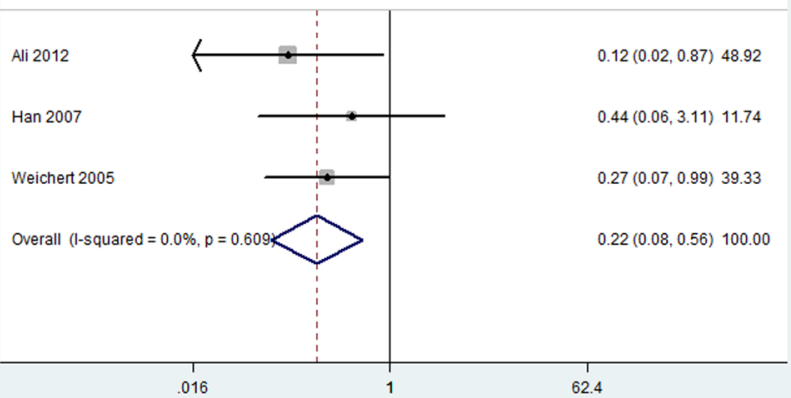

$\mathbf{F}$

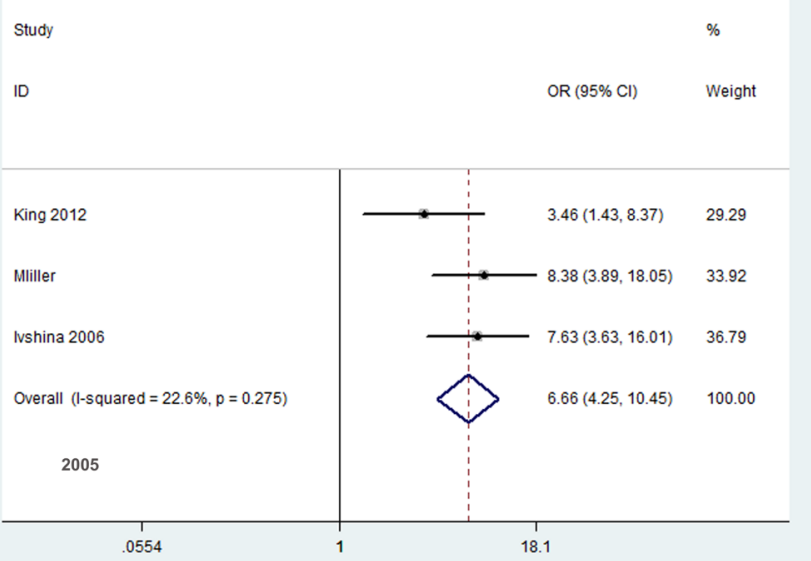

Figure 2: Forest plots of odds ratios for PLK1 expression and clinicopathological parameters in BC patients. A. Tumor size; B. Pathological grading; C. Lymph node; D. Tumor type; E. ER status; F. P53 mutation 
Begg's test did not reveal obvious evidence among all the analysis of PLK1 expression and survival (OS, $P=0.308$; CSS, $P=0.296$; DFS, $P=0.462$; Figure 5 and Table 3 ).

\section{DISCUSSION}

This meta-analysis investigating the correlation between PLK1 and BC was designed with two objectives. We first compared PLK1 expression with clinicopathological features. PLK1 expression was found to be significantly associated with tumor size, lymph node status, and pathological grading as well as with ER and p53 status, both important factors in BC. Next, we examined the prognostic value of PLK1 expression in terms of $\mathrm{BC}$ patient survival. Altogether, our results showed that PLK1 overexpression confers a strongly predictive factor for progression and prognosis in $\mathrm{BC}$.

PLK1, a key regulator of mitotic entry, is considered to be an oncology target in several tumor types, including BC [24-26]. Previous evidence demonstrated that PLK1 overexpression is closely associated with the cell cycle and peak expression of PLK1 was found to occur in the G2/M phase in in vitro models [27]. Growing bodies of evidence have revealed that alteration of PLK1 is strongly associated with aneuploidy and mitotic defects, resulting in tumorigenesis by inhibiting $\mathrm{Rb}$ and p53 genes [28]. Consistent with our results, previous studies have concluded that PLK1 negatively interacts with the ER and regulates the ER target gene in $\mathrm{BC}$ patients. Interestingly, both PLK1-co-activated and ER-targeted genes were enriched in developmental function and act as tumor suppressor factors [29], suggesting a potential interaction of PLK1 with ERs. Moreover, high PLK1 expression was positively associated with p53 mutant status, which is a potent transcription factor in tumor progression. It has been suggested that PLK1 might induce tumorigenesis by
Study

ID hr $(95 \% \mathrm{Cl})$

$\%$

Weight

\begin{tabular}{|c|c|c|c|}
\hline \multicolumn{4}{|l|}{ OS } \\
\hline Weichert 2005 & $\rightarrow$ & $2.01(0.88,4.59)$ & 24.03 \\
\hline Loddo 2009 & $\rightarrow$ & $3.46(1.37,8.71)$ & 19.16 \\
\hline Li 2011 & $\longrightarrow$ & $4.76(1.34,6.12)$ & 28.43 \\
\hline King 2012 & & $3.89(1.82,8.32)$ & 28.38 \\
\hline Subtotal $(I-$ squared $=0.0 \%, p=0.487)$ & & $3.44(2.29,5.15)$ & 100.00 \\
\hline \multicolumn{4}{|l|}{ CSS } \\
\hline Miller 2005 & $\rightarrow$ & $1.74(1.01,2.98)$ & 52.36 \\
\hline Ali 2012 & $\rightarrow$ & $2.60(1.30,5.20)$ & 31.76 \\
\hline Donizy 2016 & $\longrightarrow$ & $6.13(2.30,16.33)$ & 15.89 \\
\hline Subtotal $(\mathrm{I}$-squared $=59.5 \%, p=0.085)$ & & $2.41(1.63,3.57)$ & 100.00 \\
\hline \multicolumn{4}{|l|}{ DFS } \\
\hline Ivshina 2006 & $\rightarrow$ & $1.74(1.38,2.65)$ & 66.63 \\
\hline Loddo 2009 & & $3.31(1.57,6.97)$ & 12.77 \\
\hline Maire 2012 & & $3.41(1.03,11.26)$ & 4.96 \\
\hline King 2013 & & $6.05(2.13,17.17)$ & 6.51 \\
\hline Donizy 2016 & & $3.62(1.50,8.74)$ & 9.13 \\
\hline Subtotal $(\mathrm{I}$-squared $=52.9 \%, p=0.075)$ & & $2.26(1.73,2.95)$ & 100.00 \\
\hline $\begin{array}{c}1 \\
.0582\end{array}$ & & & \\
\hline
\end{tabular}

Figure 3: Meta-analysis comparing PLK1 expression and survival in BC patients. 
downregulating p53 [30]. In a previous study, researchers showed that PLK1 is likely to be a potential target of p53 in DNA damage and directly repressed expression by $\mathrm{p} 53$ [31] [35]. Previous evidence also revealed that PLK1 acts as a critical component of the G2/M checkpoint and was inhibited in a p53-dependent manner in tumor cells [32]. Nevertheless, the mechanism explaining how p53 status influences PLK1 expression in BC is uncertain.

Although this meta-analysis enrolled 2481 BC patients overall, several inherent limitations still exist.
First, the number of patients in the included studies (especially in OS and DSS studies) is typically small, which decreases the reliability of our results. Second, variability in detection of PLK1 expression and subsequent cut-off value selection introduces a potential source of bias. Lack of a standard threshold in practice, the cut-off value of PLK1 detection varied from $2 \%$ to $8 \%$, contributing to the potential heterogeneity. Though the semi-quantitative scale of the score (IRS) was widely used to detect the PLK1 immunohistochemical reaction
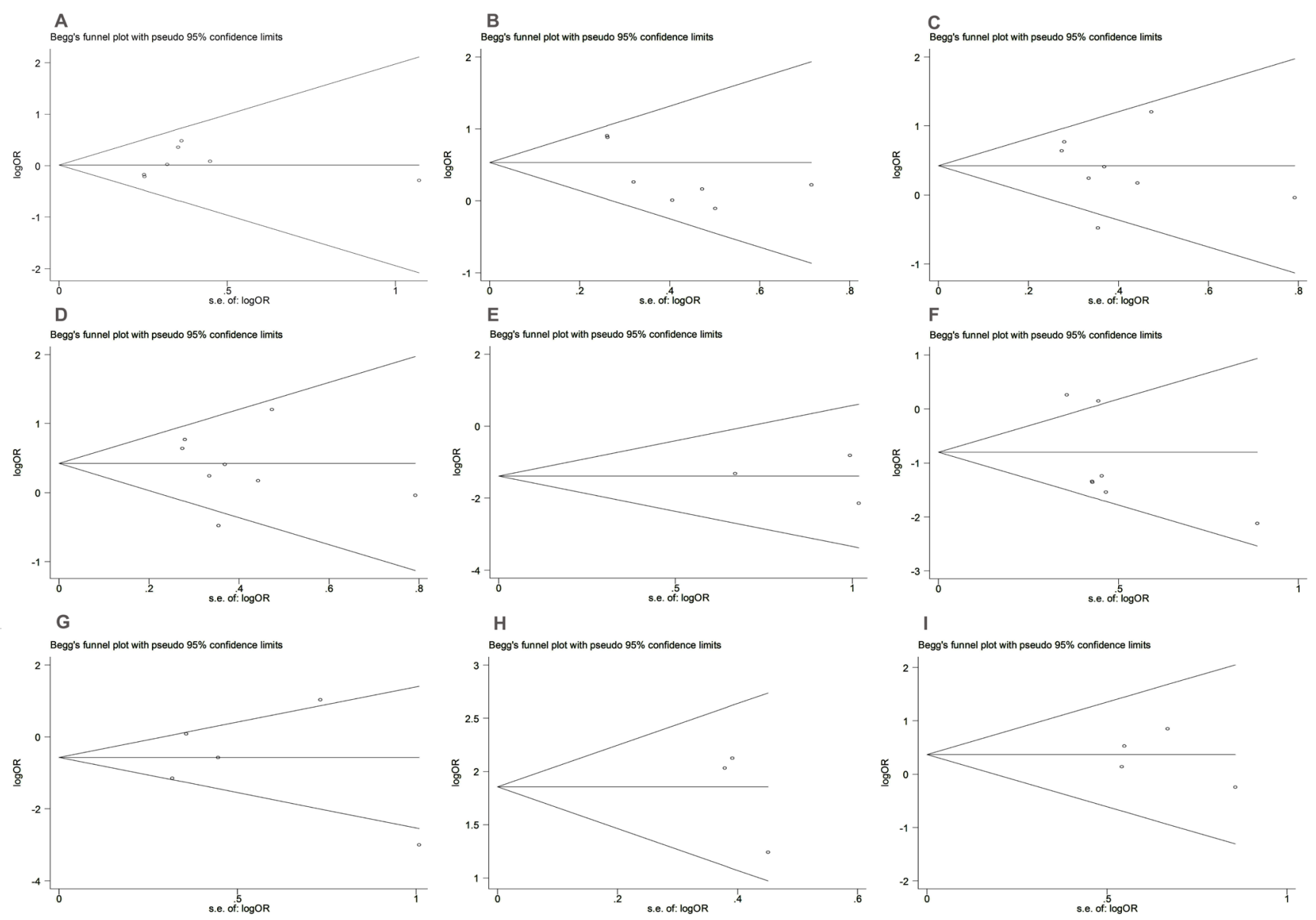

Figure 4: Funnel plot for the publication bias test of the IDH mutations and clinicopathological parameters of BC patients. A. Age; B. Tumor size; C. Pathological grading; D. Lymph node; E. Tumor type; F. ER status; G. PR status; H. P53; I. HER2.
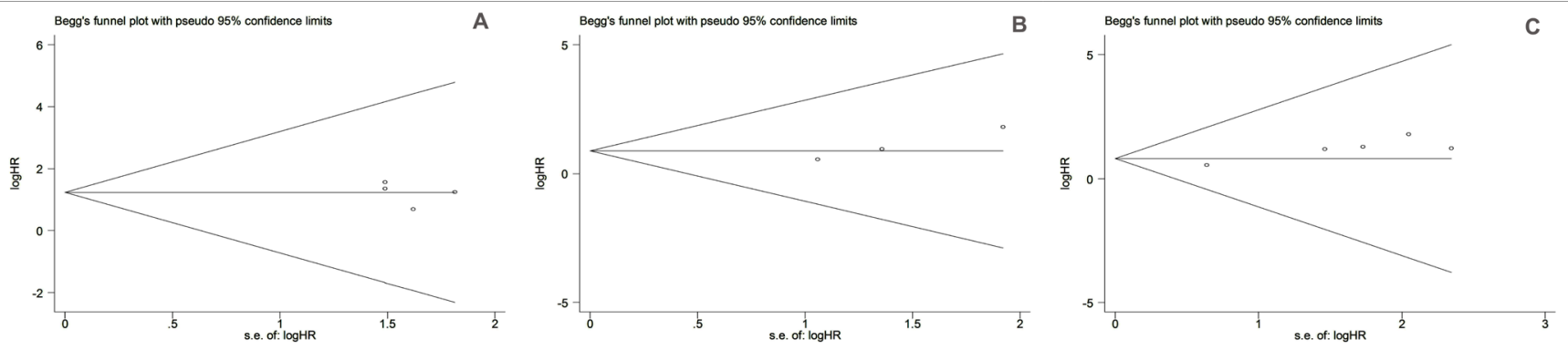

Figure 5: Funnel plots of studies evaluating the PLK1 expression and survival in BC patients A. OS; B. CSS; C. DFS 
[33], the cut-off value for high PLK1 immunoreactivity were not concordant. The high PLK1 immunoreactivity were less concordant in the included studies. King et al evaluated PLK1 overexpression by the cutoff of 3 , whereas Ali et al used a cutoff value of 1 to define high PLK1 level of BC [13, 17]. Third, several studies detected PLK1 by immunohistochemistry, while other studies used qRT-PCR. Especially, three of the included studies evaluated the PLK1 expression by using microarray profile [16, 20, 21], whereas several studies performed the immunohistochemistry to observe the PLK1 level, leading to the methodological differences. Another key limitation in our study is the method for estimating the HR from KM curves by Engauge, developing an unavoidable decrease of reliability. Fourth, only eligible English and Chinese studies were included; therefore, some qualified studies of other languages were excluded, increasing the potential biases for this meta-analysis. Additionally, several studies did not report a multivariate HR but only a univariate HR. They could not provide an independent, accurate prediction of PLK1 expression because of the multiple molecular abnormalities associated with BC.

In summary, this meta-analysis supported the conclusion that PLK1 expression confers a useful predictive factor for larger tumor size and positive lymph node status, in addition to higher tumor grading. More importantly, high PLK1 expression was closely associated with ER-positive BC and with BC having mutant P53. Of note, we also confirmed that high PLK1 expression markedly shortened OS as well as DFS and DSS for $\mathrm{BC}$ patients. However, further well-designed studies enrolled with large cohort patients are needed to define the authentic clinicopathological and prognostic value of PLK1 for BC patients.

\section{METHODS AND MATERIALS}

\section{Search strategy and selection criteria}

An electronic literature search was carried out in the PubMed, Web of Science, and Embase databases (up to 31 October 2017) by using the following MeSHs: "(polo-like kinase 1 OR PLK1 OR serine/threonineprotein kinase 13 OR STPK13) and breast cancer." Meanwhile, we also searched the eligible studies in Chinese databases, including China Biology Medicine disc (CBM), Chongqing VIP, China National Knowledge Infrastructure (CNKI), and Wan Fang Data. Additionally, we also searched the references of all the studies and bibliographies of other pertinent articles to find related articles.

The meta-analysis considered the following as inclusion criteria: (1) proven diagnosis of $\mathrm{BC}$ in humans; (2) evaluation of the relation between PLK1 expression and clinicopathological features or prognosis of $\mathrm{BC}$ patients (overall survival (OS), cancer specific survival (CSS), and disease free survival (DFS)); (3) sufficient data to evaluate the odds ratio (OR) or hazard ratio (HR) and their 95\% confidence intervals (CIs); and (4) written in Chinese or English. Exclusion criteria for this metaanalysis were the following: (1) reviews, conference abstracts, case reports, or letters; (2) insufficient data to estimate HRs or ORs and 95\% CIs; and (3) overlapping articles.

\section{Data extraction and quality assessments}

For included studies, two investigators (Yunfeng Zhang and Zhibin $\mathrm{Wu}$ ) extracted data as follows: author name, publication year, region, sample size, cut-off value, method of PLK1 detection, calculation methods for HRs, clinical features, and survival data. Thereafter, two researchers independently assessed the quality of each study according to the Newcastle-Ottawa Quality Assessment Scale (NOS) [34]. The study considered highquality must reach 6 or higher NOS score and the study with less 6 NOS score was considered as low-quality. Any controversies were arbitrated by the third researcher (Dapeng Liu).

\section{Statistical analysis}

ORs and their 95\% CIs were pooled to evaluate the association between PLK1 level and clinicopathological features including age (old versus young), tumor size ( $\geq 2 \mathrm{~cm}$ versus $<2 \mathrm{~cm}$ ), pathological grading ( $\mathrm{G} 3$ versus G1-2), tumor type (lobular BC versus ductal BC) and lymph node status (yes versus no). The correlations of PLK1-positive and ER status (positive versus negative), PR status (positive versus negative), and p53 and HER2 mutation status (mutation versus wild-type) were also estimated by the pooled ORs and their 95\% CIs. For survival analysis, we directly extracted HRs and their 95\% CIs from the articles when data was available; otherwise, we estimated the HR based on the original data by univariate cox analysis or from the Kaplan-Meier (KM) curves according to the method described by Parmar [35]. Once both multivariate and univariate HRs and 95\% CIs were provided for the same cohort of patients, we preferentially obtained HRs from the multivariate analysis due to the influence of multiple factors on the survival outcome. The HR was considered as statistically significant if it did not overlap 1. An observed HR $>1$ suggested that high PLK1 expression implied worse survival for $\mathrm{BC}$ patients. The Chi-square-based $\mathrm{Q}$ statistical test was conducted to evaluate the heterogeneity across the studies. Higgins $\mathrm{I}^{2}$ was employed to estimate the degree of heterogeneity and $\mathrm{I}^{2}$ larger than $50.0 \%$ was considered as obvious heterogeneity. The random-effects 
(DerSimonian and Laird method) were used when the $P$ value was less than 0.05 ; otherwise, the fixed-effects model (the Mantel-Haenszel method) was performed [36]. At the end, we evaluated publication bias by using Begg's rank correlation and the funnel plot [37]. A statistically significant two-way $\mathrm{P}$ value must be less than 0.05 . All statistical analyses were performed by STATA version 12.0 software (Stata Corporation, College Station, TX).

\section{ACKNOWLEDGMENTS}

This experiment was mainly supported by National Science Foundation for Young Scientists of China, grant No. 81602597 (Referred to Xin Sun). This work was also supported in part by National Natural Science Foundation of China, grant No. 81272418 (Referred to Hong Ren).

\section{CONFLICTS OF INTERESTS}

The authors declare that they have no competing interests in this study.

\section{REFERENCES}

1. Siegel RL, Miller KD, Jemal A. Cancer statistics, 2016. CA Cancer J Clin. 2016; 66:7-30.

2. Hsu RJ, Hsu YC, Chen SP, Fu CL, Yu JC, Chang FW, Chen YH, Liu JM, Ho JY, Yu CP. The triterpenoids of Hibiscus syriacus induce apoptosis and inhibit cell migration in breast cancer cells. BMC complementary and alternative medicine. 2015; 15:65.

3. Lander ES, Linton LM, Birren B, Nusbaum C, Zody MC, Baldwin J, Devon K, Dewar K, Doyle M, FitzHugh W, Funke R, Gage D, Harris K, et al. Initial sequencing and analysis of the human genome. Nature. 2001; 409:860-921.

4. Kim HI, Quan FS, Kim JE, Lee NR, Kim HJ, Jo SJ, Lee CM, Jang DS, Inn KS. Inhibition of estrogen signaling through depletion of estrogen receptor alpha by ursolic acid and betulinic acid from Prunella vulgaris var. lilacina. Biochemical and biophysical research communications. 2014; 451:282-287.

5. Ng KW, Salhimi SM, Majid AM, Chan KL. Antiangiogenic and cytotoxicity studies of some medicinal plants. Planta medica. 2010; 76:935-940.

6. Liang S, Chen Z, Jiang G, Zhou Y, Liu Q, Su Q, Wei W, Du J, Wang H. Activation of GPER suppresses migration and angiogenesis of triple negative breast cancer via inhibition of NF-kappaB/IL-6 signals. Cancer letters. 2016.

7. Lens SM, Voest EE, Medema RH. Shared and separate functions of polo-like kinases and aurora kinases in cancer. Nature reviews Cancer. 2010; 10:825-841.

8. Shaltiel IA, Krenning L, Bruinsma W, Medema RH. The same, only different - DNA damage checkpoints and their reversal throughout the cell cycle. Journal of cell science.
$2015 ; 128: 607-620$

9. Takai N, Hamanaka R, Yoshimatsu J, Miyakawa I. Pololike kinases (Plks) and cancer. Oncogene. 2005; 24:287291.

10. Yamamoto Y, Matsuyama H, Kawauchi S, Matsumoto H, Nagao K, Ohmi C, Sakano S, Furuya T, Oga A, Naito K, Sasaki K. Overexpression of polo-like kinase 1 (PLK1) and chromosomal instability in bladder cancer. Oncology. 2006; 70:231-237.

11. Otsu H, Iimori M, Ando K, Saeki H, Aishima S, Oda Y, Morita M, Matsuo K, Kitao H, Oki E, Maehara Y. Gastric Cancer Patients with High PLK1 Expression and DNA Aneuploidy Correlate with Poor Prognosis. Oncology. 2016; 91:31-40.

12. Ramani P, Nash R, Sowa-Avugrah E, Rogers C. High levels of polo-like kinase 1 and phosphorylated translationally controlled tumor protein indicate poor prognosis in neuroblastomas. Journal of neuro-oncology. 2015; 125:103111.

13. Ali HR, Dawson SJ, Blows FM, Provenzano E, Pharoah PD, Caldas C. Aurora kinase A outperforms Ki67 as a prognostic marker in ER-positive breast cancer. British journal of cancer. 2012; 106:1798-1806.

14. Donizy P, Halon A, Surowiak P, Kaczorowski M, Kozyra C, Matkowski R. Augmented expression of Polo-like kinase 1 is a strong predictor of shorter cancer-specific overall survival in early stage breast cancer at 15-year follow-up. Oncology letters. 2016; 12:1667-1674.

15. Han.S, Ma T, Tang X. Expression and clinical significance of PLK 1and PCNA in breast carcinoma. Shandong Medical Journal. 2007; :18-20.

16. Ivshina AV, George J, Senko O, Mow B, Putti TC, Smeds J, Lindahl T, Pawitan Y, Hall P, Nordgren H, Wong JE, Liu ET, Bergh J, et al. Genetic reclassification of histologic grade delineates new clinical subtypes of breast cancer. Cancer research. 2006; 66:10292-10301.

17. King SI, Purdie CA, Bray SE, Quinlan PR, Jordan LB, Thompson AM, Meek DW. Immunohistochemical detection of Polo-like kinase-1 (PLK1) in primary breast cancer is associated with TP53 mutation and poor clinical outcom. Breast cancer research. 2012; 14:R40.

18. Li Z, Luo M, Wang B, Di H. Expression of Polo-like Kinase 1 mRNA and Its Prognostic Value in Breast Cancer. Progress in Modern Biomedicine. 2011; :3442-3445+3513.

19. Loddo M, Kingsbury SR, Rashid M, Proctor I, Holt C, Young J, El-Sheikh S, Falzon M, Eward KL, Prevost T, Sainsbury R, Stoeber K, Williams GH. Cell-cycle-phase progression analysis identifies unique phenotypes of major prognostic and predictive significance in breast cancer. British journal of cancer. 2009; 100:959-970.

20. Maire V, Nemati F, Richardson M, Vincent-Salomon A, Tesson B, Rigaill G, Gravier E, Marty-Prouvost B, De Koning L, Lang G, Gentien D, Dumont A, Barillot E, et al. Polo-like kinase 1: a potential therapeutic option in 
combination with conventional chemotherapy for the management of patients with triple-negative breast cancer. Cancer research. 2013; 73:813-823.

21. Miller LD, Smeds J, George J, Vega VB, Vergara L, Ploner A, Pawitan Y, Hall P, Klaar S, Liu ET, Bergh J. An expression signature for $\mathrm{p} 53$ status in human breast cancer predicts mutation status, transcriptional effects, and patient survival. Proceedings of the National Academy of Sciences of the United States of America. 2005; 102:13550-13555.

22. Weichert W, Kristiansen G, Winzer KJ, Schmidt M, Gekeler V, Noske A, Muller BM, Niesporek S, Dietel M, Denkert C. Polo-like kinase isoforms in breast cancer: expression patterns and prognostic implications. Virchows Archiv. 2005; 446:442-450.

23. Li W. (2009). Association of CTTN, PLK1, SKP2 and PKCiota Expression in Common Tumors with Clinicopathological Parameters. Peking Union Medical College.

24. McInnes C, Wyatt MD. PLK1 as an oncology target: current status and future potential. Drug discovery today. 2011; 16:619-625.

25. Cai XP, Chen LD, Song HB, Zhang CX, Yuan ZW, Xiang ZX. PLK1 promotes epithelial-mesenchymal transition and metastasis of gastric carcinoma cells. American journal of translational research. 2016; 8:4172-4183.

26. $\mathrm{Ng} \mathrm{W}$, Shin JS, Wang B, Lee CS. Intrinsic factors do not cause overexpression of polo-like kinase 1 (PLK1) in colorectal cancer (CRC). Pathology. 2016; 48 Suppl 1:S95-s96.

27. Degenhardt Y, Lampkin T. Targeting Polo-like kinase in cancer therapy. Clinical cancer research. 2010; 16:384-389.

28. Patel H, Zich J, Serrels B, Rickman C, Hardwick KG, Frame MC, Brunton VG. Kindlin-1 regulates mitotic spindle formation by interacting with integrins and Plk-1. Nature communications. 2013; 4:2056.

29. Wierer M, Verde G, Pisano P, Molina H, Font-Mateu J, Di Croce L, Beato M. PLK1 signaling in breast cancer cells cooperates with estrogen receptor-dependent gene transcription. Cell reports. 2013; 3:2021-2032.

30. Strebhardt K. Multifaceted polo-like kinases: drug targets and antitargets for cancer therapy. Nature reviews Drug discovery. 2010; 9:643-660.

31. Liu X, Erikson RL. Polo-like kinase (Plk)1 depletion induces apoptosis in cancer cells. Proceedings of the National Academy of Sciences of the United States of America. 2003; 100:5789-5794.

32. McKenzie L, King S, Marcar L, Nicol S, Dias SS, Schumm K, Robertson P, Bourdon JC, Perkins N, Fuller-Pace F, Meek DW. p53-dependent repression of polo-like kinase-1 (PLK1). Cell cycle (Georgetown, Tex). 2010; 9:4200-4212.

33. Halon A, Donizy P, Surowiak P, Matkowski R. ERM/Rho protein expression in ductal breast cancer: a 15 year followup. Cellular oncology (Dordrecht). 2013; 36:181-190.

34. Wells G, Shea B, O'connell D, Peterson J, Welch V, Losos M, Tugwell P. (2000). The Newcastle-Ottawa Scale (NOS) for assessing the quality of nonrandomised studies in metaanalyses.

35. Parmar MK, Torri V, Stewart L. Extracting summary statistics to perform meta-analyses of the published literature for survival endpoints. Statistics in medicine. $1998 ; 17: 2815-2834$

36. Higgins JP, Thompson SG. Quantifying heterogeneity in a meta-analysis. Statistics in medicine. 2002; 21:1539-1558.

37. Song F, Gilbody S. Bias in meta-analysis detected by a simple, graphical test. Increase in studies of publication bias coincided with increasing use of meta-analysis. BMJ. 1998; 316:471. 